\title{
EXTRAÇÃO ASSISTIDA POR ULTRASSOM E EM SOXHLET DE ÓLEO DE SEMENTES DE UVA BORDÔ E CABERNET SAUVIGNON - COMPOSIÇÃO EM ÁCIDOS GRAXOS
}

\author{
F. LIU ${ }^{1}$, J.P.V. CESARO ${ }^{1}$, A. DIÓRIO ${ }^{1}$, N. C. PEREIRA ${ }^{1}$ e O. C. M. LIMA ${ }^{1}$ \\ ${ }^{1}$ Universidade Estadual de Maringá, Departamento de Engenharia Química \\ *E-mail para contato: dandaliu@ hotmail.com
}

\begin{abstract}
RESUMO - O presente trabalho teve como objetivo avaliar possíveis alterações na composição de ácidos graxos nos óleos de sementes de uva Cabernet Sauvignon e Bordô extraídos com auxílio de ultrassom em relação à extração em Soxhlet. Para tanto, sementes de uva Cabernet Sauvignon e Bordô, secas e moídas, foram submetidas à extração, cujos teores de óleo constatados apresentaram valores na faixa de 13-14\% para o método Soxhlet. Já o processo auxiliado por ultrassom apresentou valores de teor de óleo na faixa de 10-11\%, para ambas as variedades de sementes, confirmando a eficiência deste método de extração quando comparado ao Soxhlet. Com relação à composição de ácidos graxos, observou-se que o ácido linoleico foi majoritário, apresentando concentração na faixa de 629,52 - 676,99 mg/góleo, sendo que também foi verificada a presença mais expressiva de ácidos graxos saturados nos extratos submetidos ao processo de extração auxiliada por ultrassom, evidenciando assim, a instabilidade das insaturações rompidas, geralmente na presença das ondas ultrassônicas.
\end{abstract}

\section{INTRODUÇÃO}

Para os processos viníferos são destinados, aproximadamente, $71 \%$ da produção mundial de uva (Demiral e Ayan, 2011), sendo o bagaço de uva um dos resíduos sólidos gerados, os quais se tornam um problema ambiental, devido à sua grande produção e baixo aproveitamento (Fiori et al., 2014). O bagaço é majoritariamente composto por sementes de uva, as quais constituem matéria prima na extração do óleo de sementes de uva. Neste óleo encontram-se componentes orgânicos, como ácidos graxos, dentre os quais o ácido linoleico, que se apresenta como o mais abundante e, por possuir relevância na redução de distúrbios cardiovasculares, mostra-se muito benéfico ao organismo humano (Davidov-Pardo e Mcclements, 2015).

Dessa forma, a extração do óleo de sementes de uva é desejável, pois este óleo apresenta grande potencial alimentício e poderá, também, ter grande interesse comercial. Considera-se como metodologia tradicional de extração o uso de solventes, comumente o hexano, compondo parte fundamental dos processos produtivos farmacológicos, cosméticos e alimentícios, porém, apresenta elevado custo de capital, riscos para a saúde humana e contaminação ambiental (Rabrenović et al., 2014). Na busca por tecnologias verdes e econômicas, encontra-se a extração assistida por ondas ultrassônicas, sendo esta utilizada na obtenção de compostos de matrizes vegetais (Rombaut et al., 2014). Esta técnica de extração 
objetiva reduzir o consumo de energia, apresentando eficiência semelhante e menor tempo de operação, quando comparada ao método de extração em Soxhlet. Sendo assim, é possível avaliar a influência de diferentes técnicas de extração na composição em ácidos graxos do óleo de sementes de uva.

\section{MATERIAIS E MÉTODOS}

\subsection{Materiais}

Foram doados pela empresa Vinícola Toledo (Toledo - PR), $100 \mathrm{~kg}$ de sementes de uva Cabernet Sauvignon, safra 2011.As sementes de uva Bordô, foram fornecidas pela empresa Vinícola Vinhos Randon Ltda. (Pinheiro Preto - SC), $100 \mathrm{~kg}$, safra 2011. Estas sementes foram armazenadas em freezer para manter as propriedades e características organolépticas. Após a moagem e peneiramento o diâmetro de Sauter para as sementes de uva Cabernet Sauvignon moídas foi de $8 \cdot 10^{-2} \mathrm{~cm}$ e para as sementes de uva Bordô foi de $8,48 \cdot 10^{-2} \mathrm{~cm}$.

\subsection{Métodos}

Foram definidos os métodos de extração do óleo das sementes de uva Cabernet Sauvignon e Bordô pelas técnicas Soxhlet e ultrassom, com posterior caracterização da composição em ácidos graxos do óleo obtido.

\subsection{Processo de Extração}

Extração em Soxhlet: os experimentos de extração por Soxhlet foram realizados em duplicata, utilizando-se 10 gramas de sementes, previamente limpas, moídas e secas a $80^{\circ} \mathrm{C}$. O solvente utilizado foi o hexano, com um volume de $300 \mathrm{~mL}$ em cada experimento e cujo ponto de ebulição $\left(68^{\circ} \mathrm{C}\right)$ determinou a temperatura de extração. Os procedimentos para se obter o teor de óleo foram realizados nos tempos de 2, 6, 10 e 20 horas, além do esgotamento em 48 horas. Para o cálculo do teor de óleo foi utilizada a Equação 1.

$$
\text { Teor de óleo }(\%)=\frac{m_{\text {óleo }}}{m_{\text {semente }}} .100
$$

Em que $m_{\text {semente }}$ é a massa de sementes de uva limpas e secas, utilizadas em cada extração e $m_{\text {óleo }}$ é a massa de óleo obtido após extração.

Extração auxiliada por ultrassom: o solvente foi hexano em contato com 10 gramas de sementes secas e moídas. A lavadora ultrassônica Schuster Modelo L200 operou na frequência de $42 \mathrm{kHz}$ e os parâmetros foram a temperatura, o tempo e a razão mássica (sementes/solvente) previamente definida por meio de um planejamento experimental, tendo sido escolhido o Box-Behnken. O tempo foi de 30, 60 e 90 minutos; razões mássicas de 1:4, $1: 6,1: 8$ e temperaturas de 30,40 e $50{ }^{\circ} \mathrm{C}$.

\subsection{Composição em Ácidos Graxos Totais}

A composição em ácidos graxos do óleo de sementes de uva, expressa em ésteres metílicos, foi determinada por meio de cromatografia gasosa com detectores de chama, que é o método mais preciso (Sahena et al., 2009), visto que os ésteres metílicos possuem a 
temperatura de volatilidade mais amena, gerando, assim, um pico de cromatografia mais nítido para os ácidos graxos mais comuns (Sahena et al., 2009; Brunner, 2005).

Para a derivatização, em tubo de ensaio, foram aferidos $100 \mathrm{mg}$ do óleo, adicionou-se $2,0 \mathrm{~mL}$ de n-heptano seguindo agitação até a completa solubilização do material. Na sequência foram adicionados $2,0 \mathrm{~mL}$ de solução $2 \mathrm{~mol} / \mathrm{L}$ de hidróxido de potássio em metanol com nova agitação durante cinco minutos. Quando as duas fases formadas foram completamente separadas, utilizou-se o sobrenadante para injeção no cromatógrafo a gás da Thermo, modelo Trace Ultra 3300, com detector de ionização de chama e coluna capilar de sílica fundida CP - 7420 (Select FAME, $100 \mathrm{~m}$ x 0,25 mm e 0,25 $\mu \mathrm{m}$ de cianopropil). O gás de arraste utilizado foi Hélio em razão slit 1:10. O processo de análise teve inicio na temperatura de $140{ }^{\circ} \mathrm{C}$ e rampa de aquecimento de $5{ }^{\circ} \mathrm{C} / \mathrm{min}$ com máximo em $250{ }^{\circ} \mathrm{C}$. O injetor e o detector foram mantidos na temperatura de $260{ }^{\circ} \mathrm{C}$ e $220^{\circ} \mathrm{C}$, respectivamente.

\section{RESULTADOS E DISCUSSÃO}

\subsection{Processos de Extração}

Extração por Soxhlet: os teores de óleo obtidos na extração em Soxhlet para as sementes de uva Cabernet Sauvignon e Bordô, estão apresentados na Figura 1.

Figura 1. Teor de óleo obtido em Soxhlet, para as sementes das variedades Cabernet Sauvignon e Bordô.



Foi possível analisar, pela Figura 1, que os maiores valores para o teor de óleo obtido ocorreram no tempo de 48 horas, isto é, no maior tempo de extração, para ambas as variedades de sementes. Os maiores teores foram de, aproximadamente, 14\% e 13\% para as sementes de Cabernet Sauvignon e Bordô, respectivamente.

Extração auxiliada por ultrassom: foi determinada a eficiência do processo de extração auxiliada por ultrassom, para as sementes de uva Cabernet Sauvignon e Bordô, cujos dados 
foram apresentados na Tabela 1. Considerou-se eficiência de $100 \%$ a quantidade de óleo obtida pelo método de extração Soxhlet, na condição de esgotamento extrativo, com 48 horas de duração.

Tabela 1. Eficiência obtida na extração auxiliada por ultrassom das sementes de uva Cabernet Sauvignon e Bordô

\begin{tabular}{cccccc}
\hline Experimento & Tempo (min) & T $\left({ }^{\circ} \mathbf{C}\right)$ & Razão $(\mathbf{m} / \mathbf{m})$ & Eficiência $^{\mathbf{a}} \mathbf{C S}(\%)$ & Eficiência $^{\mathbf{a}} \mathbf{B D}(\%)$ \\
\hline 1 & 30 & 30 & $1: 6$ & $56,67 \pm 1,83$ & $55,81 \pm 1,30$ \\
2 & 90 & 30 & $1: 6$ & $65,98 \pm 0,91$ & $64,18 \pm 1,46$ \\
3 & 30 & 50 & $1: 6$ & $65,67 \pm 0,99$ & $74,25 \pm 0,63$ \\
4 & 90 & 50 & $1: 6$ & $76,32 \pm 2,42$ & $81,76 \pm 1,62$ \\
5 & 30 & 40 & $1: 4$ & $70,42 \pm 3,32$ & $59,24 \pm 1,13$ \\
6 & 90 & 40 & $1: 4$ & $77,80 \pm 3,47$ & $64,80 \pm 1,18$ \\
7 & 30 & 40 & $1: 8$ & $80,46 \pm 3,13$ & $73,07 \pm 0,17$ \\
8 & 90 & 40 & $1: 8$ & $72,06 \pm 1,88$ & $73,60 \pm 3,08$ \\
9 & 60 & 30 & $1: 4$ & $69,28 \pm 2,23$ & $55,70 \pm 1,01$ \\
10 & 60 & 50 & $1: 4$ & $72,74 \pm 1,19$ & $62,49 \pm 0,20$ \\
11 & 60 & 30 & $1: 8$ & $68,63 \pm 0,77$ & $65,78 \pm 0,05$ \\
12 & 60 & 50 & $1: 8$ & $79,00 \pm 0,92$ & $66,02 \pm 1,69$ \\
13 & 60 & 40 & $1: 6$ & $67,47 \pm 1,40$ & $58,64 \pm 1,61$ \\
14 & 60 & 40 & $1: 6$ & $68,17 \pm 1,79$ & $58,92 \pm 1,68$ \\
15 & 60 & 40 & $1: 6$ & $68,21 \pm 0,45$ & $53,74 \pm 0,99$ \\
\hline
\end{tabular}

${ }^{a}$ média seguida de desvio padrão

Observou-se que, para as sementes de Cabernet Sauvignon, a maior eficiência ocorreu no experimento 7 , com condições de 30 minutos, $40{ }^{\circ} \mathrm{C}$ e razão mássica $1: 8$. Eficiências semelhantes foram obtidas para os experimentos $4,6,8,10$ e 12, nos quais o tempo de extração foi maior, a temperatura manteve-se idêntica ou sofreu aumento, porém a razão mássica foi reduzida. Já para as sementes de uva Bordô, a maior eficiência registrada ocorreu no experimento 4, cujas condições de temperatura e tempo foram mais intensas do que aquelas aplicadas às sementes de CS do experimento 7. Nos casos 3,7 e 8 também foram registradas eficiências superiores a $70 \%$ para a variedade Bordô, contudo, para quase todos os casos, os rendimentos desta variedade se apresentaram mais baixos quando comparados aos das sementes de CS. Por fim, verificou-se que, tanto a extração por Soxhlet quanto a auxiliada por ultrassom apresentaram teores de óleo dentro dos limites encontrados na literatura, tais como os resultados de Beveridge et al. (2005), variando de 6,6 a 11,1\% para éter de petróleo e Luque-Rodriguez et al. (2005), de 9,6 a 13,9\% com hexano.

\subsection{Composição em Ácidos Graxos}

Para os experimentos em ultrassom com maior rendimento, isto é, caso 7 para as sementes de uva Cabernet Sauvignon e caso 4 para a Bordô, bem como para o óleo do esgotamento em 48 horas de extração Soxhlet para ambas as variedades de sementes, foram obtidas as composições em ácidos graxos, cujos dados foram dispostos na Tabela 2. 
Tabela 2. Composição em ácidos graxos (mg/góleo), obtida por cromatografia gasosa dos extratos de sementes de uva

\begin{tabular}{|c|c|c|c|c|}
\hline \multirow{2}{*}{ Ácidos Graxos } & \multicolumn{2}{|c|}{ Cabernet Sauvignon } & \multicolumn{2}{|c|}{ Bordô } \\
\hline & Soxhlet & Ultrassom & Soxhlet & Ultrassom \\
\hline 14:0 (mirístico) & $1,06^{\mathrm{a}} \pm 0,09$ & $1,21^{\mathrm{b}} \pm 0,01$ & $0,79^{a} \pm 0,03$ & $0,95^{\mathrm{b}} \pm 0,07$ \\
\hline 16:0 (palmítico) & $64,21^{\mathrm{a}} \pm 2,7$ & $86,99^{\mathrm{a}} \pm 2,87$ & $64,15^{\mathrm{a}} \pm 1,09$ & $75,6^{\mathrm{a}} \pm 0,46$ \\
\hline 16:1n-7 (palmitoleico) & $1,23^{\mathrm{a}} \pm 0,18$ & $1,7^{\mathrm{a}} \pm 0,49$ & $1,61^{\mathrm{a}} \pm 0,34$ & $1,98^{\mathrm{a}} \pm 0,15$ \\
\hline 17:0 (heptadecanoico) & $0,69^{\mathrm{a}} \pm 0,15$ & $0,88^{b} \pm 0,02$ & $0,45^{\mathrm{a}} \pm 0,01$ & $0,72^{\mathrm{a}} \pm 0,14$ \\
\hline 18:0 (esteárico) & $33,12^{\mathrm{a}} \pm 1,39$ & $41,47^{\mathrm{a}} \pm 0,56$ & $34,35 \mathrm{a} \pm 2,09$ & $32,28^{\mathrm{ab}} \pm 0,16$ \\
\hline 18:1n-9c (oleico) & $165,8^{a} \pm 1,94$ & $144,31^{\mathrm{a}} \pm 0,03$ & $172,31^{\mathrm{a}} \pm 1,82$ & $178,18^{\mathrm{ab}} \pm 1,65$ \\
\hline 18:1n-7 (cis-vaccênico) & $3,4^{\mathrm{a}} \pm 0,53$ & $2,47^{b} \pm 0,11$ & $3,01^{\mathrm{a}} \pm 0,61$ & $3,06^{\mathrm{ab}} \pm 0,62$ \\
\hline 18:2n-6 (linoleico) & $672,52^{\mathrm{b}} \pm 4,86$ & $629,41^{\mathrm{b}} \pm 8,25$ & $676,99^{\mathrm{a}} \pm 0,06$ & $632,82^{\mathrm{a}} \pm 0,97$ \\
\hline 18:3n-3 ( $\alpha$-linolênico) & $4,2^{\mathrm{a}} \pm 0,3$ & $4,07^{\mathrm{a}} \pm 0,05$ & $4,29^{\mathrm{a}} \pm 0,19$ & $4,46^{\mathrm{a}} \pm 0,31$ \\
\hline 20:0 (araquídico) & $1,69^{\mathrm{a}} \pm 0,08$ & $2,24^{\mathrm{a}} \pm 0,03$ & $1,54^{\mathrm{a}} \pm 0$ & $1,56^{\mathrm{a}} \pm 0$ \\
\hline 20:1n-9 (11-eicosenoico) & $1,21^{\mathrm{a}} \pm 0,15$ & $2,04^{\mathrm{a}} \pm 0,1$ & $1,38^{\mathrm{a}} \pm 0,14$ & $1,73^{\mathrm{ab}} \pm 0,13$ \\
\hline 22:0 (behênico) & $0,47^{\mathrm{b}} \pm 0$ & $0,79^{\mathrm{a}} \pm 0,21$ & $0,41^{\mathrm{b}} \pm 0,05$ & $0,44^{\mathrm{a}} \pm 0,08$ \\
\hline 23:0 (tricosanóico) & $17,96^{\mathrm{a}} \pm 2,79$ & $11,03^{\mathrm{a}} \pm 0,11$ & $22,06^{\mathrm{b}} \pm 2,06$ & $11,7^{\mathrm{a}} \pm 0,37$ \\
\hline 24:0 (lignocérico) & Não detectado & Não detectado & $0,41 \pm 0,19$ & $0,25 \pm 0$ \\
\hline$\Sigma$ Saturados & $101,24 \pm 2,67$ & $133,58 \pm 1,49$ & $102,1 \pm 2,45$ & $111,81 \pm 0,64$ \\
\hline$\Sigma$ Monoinsaturados & $171,64 \pm 1,98$ & $150,52 \pm 0,22$ & $178,32 \pm 1,86$ & $184,94 \pm 1,4$ \\
\hline$\Sigma$ Poli-insaturados & $676,72 \pm 3,65$ & $633,48 \pm 5,87$ & $681,29 \pm 0,18$ & $637,28 \pm 0,47$ \\
\hline$\Sigma \mathrm{n}-6$ & $672,52 \pm 3,44$ & $629,41 \pm 5,83$ & $676,99 \pm 0,04$ & $632,82 \pm 0,68$ \\
\hline$\Sigma \mathrm{n}-3$ & $4,2 \pm 0,21$ & $4,07 \pm 0,04$ & $4,29^{\mathrm{a}} \pm 0,14$ & $4,46^{\mathrm{a}} \pm 0,22$ \\
\hline$n-6 / n-3$ & $160,42 \pm 7,31$ & $154,71 \pm 0,05$ & $157,89 \pm 4,99$ & $142,27 \pm 7,09$ \\
\hline
\end{tabular}

As médias seguidas de mesma letra superescrita nas linhas não diferes estatisticamente entre si pelo teste de Tukey ao nível de 5\% de probabilidade.

Pela Tabela 2, verificou-se que os teores em ácidos graxos mais expressivos para o óleo de Cabernet Sauvignon e Bordô, para ambos os métodos de extração, foram o ácido linoleico, oleico, palmítico e esteárico. Em menores quantidades, foram constatados os ácidos $\alpha$ lonolênico, aracdônico, eicosenoico, palmitoleico, mirístico, behênico e lignocérico, sendo este último presente somente no óleo da variedade Bordô. Não obstante, ao se comparar os dois métodos de extração, observou-se que, na maioria dos casos, a presença de ácidos graxos saturados foi mais expressiva com o uso do ultrassom, ao passo que, em Soxhlet, foram os insaturados. Possivelmente, as insaturações (mais instáveis do que ligações sigma pelo fato de possuírem ligações pi), na presença de ondas ultrassônicas se rompem, justificando, por conseguinte, a presença mais expressiva de ácidos graxos saturados nos extratos obtidos pelo método auxiliado por ultrassom. Por fim, notou-se também que o ácido linoleico, majoritário, apresentou concentração na faixa de 630 - $677 \mathrm{mg} / \mathrm{g}_{\text {óleo, }}$ sendo este o principal ácido graxo responsável pelo controle e redução do mau colesterol (LDL-C) (Fernández et al., 2013).

\section{CONCLUSÕES}

Concluiu-se que, para as sementes de uva Cabernet Sauvignon e Bordô os teores de óleo obtidos pelo método de extração por Soxhlet foram de $14 \%$ e 13\%, respectivamente, contudo, a técnica de extração auxiliada por ultrassom apresentou maior eficiência, demonstrando maior eficácia desse processo quando comparada à técnica Soxhlet, por demandar condições operacionais menos intensas, gerando resultados semelhantes. Não obstante, com relação ao perfil de ácidos graxos, verificou-se que as insaturações, por se tratarem de ligações instáveis, 
sofreram rupturas quando os extratos de ambas as variedades de sementes foram submetidos ao método de extração auxiliada por ultrassom, gerando, por conseguinte, maior quantidade de ácidos graxos saturados constatados após a aplicação desse método extrativo. $\mathrm{O}$ ácido linoleico apresentou concentração na faixa de 629,52 - 676,99 mg/góleo, demonstrando-se majoritário.

\section{AGRADECIMENTOS}

Os autores agradecem o financiamento fornecido pela CAPES ao projeto.

\section{REFERÊNCIAS BIBLIOGRÁFICAS}

BEVERIDGE, T.H.; GIRARD, B.; KOPP, T.; DROVER, J.C.G. Yield and composition of grape seed oils extracted by supercritical carbon dioxide and petroleum ether: varietal effects. Journal of Agricultural and Food Chemistry. v. 53, p. 1799-1804, 2005.

BRUNNER, G. Supercritical fluids: technology and application to food processing. Journal of Food Engineering v.67, p. 21-33, 2005.

DAVIDOV-PARDO, G.; DAVID, J. M. Nutraceutical delivery systems: Resveratrol encapsulation in grape seed oil nanoemulsions formed by spontaneous emulsification. Food Chemistry, v.167, p.205-212, 2015.

DEMIRAL, I.; AYAN, E.A.; Pyrolysis of grape bagasse: effect of pyrolysis conditions on the product yields and characterization of the liquid product. Bioresources Technology, v. 102, p.3946-3951, 2011.

FERNÁNDEZ, L.; CASAL, S.; CRUZ, R.; PEREIRA, J.A.; RAMALHOSA, E. Seeds of ten traditional Portuguese grape varieties with interesting chemical and antioxidant properties. Food Research International, v. 50, p. 161-166, 2013.

FIORI, L.; LAVELLI, V.; DUBA, K. S.; HARSHA, P. S. C. S.; MOHAMED, H. B.; GUELLA, G. Supercritical CO2 extraction of oil from seeds of six grape cultivars: Modeling of mass transfer kinetics and evaluation of lipid profiles and tocol contents. Journal of Supercritical Fluids, v.94, p.71-80, 2014.

LUQUE-RODRIGUEZ, J.M.; CASTRO, M.D.L; PÉREZ-JUAN, P. Extraction of fatty acids from grape seed by superheated hexane. Talanta v. 68, n. 1, p.126-130, 2005.

RABRENOVIĆ, B. B., DIMIĆ, E. B., NOVAKOVIĆ, M. M., TESSEVIĆ, V. V., BASIĆ, Z. $\mathrm{N}$. The most important bioactive components of cold pressed oil from different pumpkin (Cucurbita pepo L.) seeds. Food Science and Technology, v.55, p.521-527, 2014.

ROMBAUT, N., SAVOIRE, R., THOMASSET, B., BÉlliARD, T., CASTEllo, J., HECKE, É. V., LANOISELLÉ, J. Grape seed oil extraction: interest of supercritical fluid extraction and gas-assisted mechanical extraction for enhancing polyphenol coextraction in oil. Comptes Rendus Chimie, v.17, p.284-292, 2014.

SAHENA, F.; ZAIDUL, I.S.M.; JINAP, S.; KARIM, A.A.; ABBAS, K.A.; NOULAINI, N.A.N.; OMAR, A. K. M. Application of supercritical CO2 in lipid extraction - A review. Journal of Food Engineering. v.95, p. 240-253, 2009. 